\title{
Le sacré au prisme de la muséologie
}

\section{François Mairesse}

\section{OpenEdition}

\section{Journals}

Édition électronique

URL : http://journals.openedition.org/iss/1293

DOI : $10.4000 /$ iss. 1293

ISSN : 2306-416

\section{Éditeur}

ICOM - International Council of Museums

\section{Édition imprimée}

Date de publication : 12 octobre 2019

Pagination : 23-30

ISBN : 978-92-9012-467-2

ISSN : 2309-1290

\section{Référence électronique}

François Mairesse, « Le sacré au prisme de la muséologie », ICOFOM Study Series [En ligne], 47(1-2) 2019, mis en ligne le 12 octobre 2019, consulté le 24 septembre 2020. URL : http:// journals.openedition.org/iss/1293; DOI : https://doi.org/10.4000/iss.1293 


\section{Le sacré au prisme de la muséologie}

\section{François Mairesse Sorbonne nouvelle-Paris3, CERLIS, CNRS, ICCA}

Les articles rassemblés dans ce numéro autour des liens entre musées, muséologie et sacré, ont été pour la plupart nourris par les discussions du $4 \mathrm{I}^{\mathrm{e} m e}$ symposium organisé par l'ICOFOM autour de cette thématique à Téhéran, du I5 au I9 octobre 2018 (Mairesse, 20I8). Ces contributions cherchent à poursuivre les réflexions et les discussions souvent passionnées, entamées lors de ces rencontres.

Nul doute que la thématique du sacré soit d'une importance capitale pour le monde des musées, tant par les objets que ces derniers présentent que par l'essence-même de l'institution et le rôle parfois ambigu que cette dernière joue à cet égard, le musée pouvant être à la fois accusé de sacraliser des objets profanes et de profaner des objets sacrés. D'un point de vue étymologique, le terme comprend l'idée de séparation (Texier, I990) : l'espace du sacré est lié à cette expérience particulière de la rencontre du divin (hiérophanie). Crainte et effroi (Otto, I969), expérience de la réalité ou de ce qu'il y a de plus réel dans ce monde (Eliade, 1965). Si nous définissons la muséologie, comme l'a suggéré Stránský dès les années i980, comme l'étude d’une relation spécifique de l'homme à la réalité (Gregorová, I980), de quelle réalité parlons-nous? D'emblée, le contexte dans lequel écrit Stránský nous permet d'avoir une certaine idée de ses conceptions en la matière, issues des Lumières et de la pensée marxiste. Lorsque le muséologue de Brno envisage cette perspective spécifique, c'est à partir d'une vision fondée sur la constitution active de collections matérielles en lien avec les disciplines classiques de l'archéologie, l'ethnographie, les sciences naturelles ou l'histoire de l'art (Mairesse, 2019). Le rôle du musée, selon cette vision des choses, s'inscrit dans une approche scientifique (et accessoirement esthétique) du monde. Le sacré y apparaît essentiellement comme un objet d'étude ethnographique ou historique à partir d'une perspective largement 
agnostique ou athée : toute autre type d'expérience que celui proposé par la science ou l'esthétique y semble quelque peu saugrenu, à moins d'être à son tour observée et analysée de la même manière.

Pour autant, le monde muséal, en cherchant à se défaire de la dimension religieuse omniprésente dans le patrimoine dont il a la charge, ne semble avoir fait que déplacer le curseur. Son mode de fonctionnement comporte aussi, à travers ses pratiques et ses espaces, une dimension se rapprochant de celle que l'on observe au sein d'autres lieux sacrés : architecture, modes de visite (Duncan et Wallach, 1978), positionnement des conservateurs-initiés par rapport aux profanes, etc. (Mairesse, 2014). Parallèlement à ces évolutions que l'on peut suivre au gré de l'histoire muséale, les territoires du sacré (temples, églises, lieux de pèlerinage) ont également développé des dispositifs d'exposition dont l'utilisation apparaît étonnamment similaire à celle de musées classiques : visites, vente de catalogues ou de souvenirs, etc. Parallèlement, nombre de lieux anciennement sacrés (temples, églises, etc.) sont devenus à la fois musées et lieux de patrimoine.

Que peut-on dire de cette étroite imbrication entre deux mondes qu'a priori tout sépare, mais dont l'analyse révèle des pratiques parfois étonnamment similaires? Les conclusions du colloque de Téhéran, aussi bien que les articles de ce numéro qui en poursuivent la discussion, n'ont pas pour objectif de circonscrire le sujet, mais permettent cependant de présenter quelques perspectives de recherche afin de mieux comprendre l'interrelation entre les deux domaines.

\section{Le stade du sacré}

Un premier élément ayant assez directement émergé des discussions concerne la nature du sacré, sa présence à travers certains objets et la hiérarchie implicite qui en découle. Une perspective assez générale, largement évoquée durant le colloque, vise en effet à reconnaître le sacré dans un très grand nombre de lieux ou d'objets : paysages, forêts, cours d'eau, montagnes, objets de culte, etc. Selon une telle vision des choses, le sacré apparait inévitablement aussi bien au sein du musée qu'au détour d'un sentier, dans la nature. Cependant, au même titre que d'autres hiérarchies (esthétique, valeur financière), certains lieux ou certains objets, comme la Kaaba ou le Saint Suaire de Turin, apparaissent plus sacrés que d'autres. Si l'on peut envisager les œuvres d'art ou des témoignages historiques sous l'angle d'une certaine sacralité, les objets de culte présentent pour la plupart un « niveau de sacralité » différent de celui que l'on pourrait retrouver devant la Pierre de Rosette ou même la Joconde, pour ne citer que deux exemples célébres. Cet état de fait peut apparaître trivial ; il permet néanmoins de remarquer que les objets considérés actuellement comme les plus sacrés (toutes sociétés confondues) ne sont pas conservés dans des musées (du moins des musées laïques, certains temples ayant aussi créé des espaces muséaux). Si l'on peut observer de près la momie de Toutankhamon, assurément d'un très haut niveau de sacralité durant l'époque pharaonique, les objets ou les lieux les plus sacrés des grandes religions actuelles (reliques, espaces du sacré) 


\section{Introduction}

ne figurent pas dans les musées mais demeurent liés au culte. Le sacré apparaît encore, dans cette perspective, comme retiré des lieux du profane, et même si le musée fonctionne largement comme un espace en dehors du quotidien, il ne semble pas offrir de caractéristiques similaires à celles qui prévalent dans des lieux directement conçus à partir du rapport traditionnel au sacré. Le musée cherche à muséaliser le monde à travers les objets, mais certains d'entre eux semblent encore avoir suffisamment de force pour lui résister. Si l'on poursuit ce raisonnement, il convient de remarquer que la force des peuples ou des sociétés associées à certains objets détermine leur place (ou leur absence) au sein du musée : au plus un culte s'affaiblit (ou est lié à un pouvoir faible sur les plans politiques et militaires), au plus il a de chance d'être muséalisé. Il en va ainsi du patrimoine des sociétés disparues, dominées ou colonisées. A l'inverse, les demandes de restitution adressées (et obtenues) depuis quelques années par des peuples autochtones et sociétés autrefois colonisées, témoignent à la fois de nouveaux rapports de force géopolitiques et du regain de vitalité de certaines croyances ancestrales.

Un second principe de notre relation au sacré est constitué par le lien entre le lieu (ou l'objet) et l'intercesseur, ce dernier reconnaissant ou faisant jaillir le sacré. Pour que ce dernier se manifeste, il faut un sorcier, un ecclésiastique ou à tout le moins un acteur reconnu par la communauté et habilité à réunir les deux mondes (profane et sacré), soit en attestant de sa présence, soit en la faisant apparaître par le biais d'un rituel. La pratique muséale, dans cette perspective, diffère très largement de celle que l'on retrouve dans les lieux de culte. Certes, le conservateur (ou un régisseur, un médiateur, selon les principes de la hiérarchie muséale) atteste, en quelque sorte, de la muséalité d'un objet, c'est-à-dire de ce qui a conditionné sa sélection, sa thésaurisation et sa présentation : une chaise muséalisée perd son statut de chaise dans le monde profane (soumis à sa valeur d'usage) pour être présentée en fonction de sa valeur de documentation et de représentation du réel (sa muséalité). Conservateurs et membres du clergé sont donc tous les deux considérés comme habilités à reconnaître un certain niveau de réalité échappant au monde profane. En revanche, le pouvoir du conservateur s'arrête là où commence réellement celui du prêtre ou du shaman, qui peut faire jaillir le sacré ou entrer en communion avec la transcendance. Le plan de la muséalité, dans cette perspective, apparaît en décalage avec celui auquel mène le sacré, le rôle du musée et celui de l'église ne pouvant mener, malgré quelques similitudes dans leur approche, aux mêmes perspectives sur le Réel.

Si, comme cela a déjà été suggéré, il est possible d'évoquer les multiples ressemblances entre musées et temples (Mairesse, 2014), il est tout aussi possible de chercher à comprendre en quoi les dispositifs de thésaurisation et de présentation utilisés par les deux institutions diffèrent. La structure de présentation du sacré, en ce sens, constitue un troisième axe de réflexion. Musées et temples (églises, mosquées, etc.) montrent des objets - reliques, objets de culte, témoignages historiques - selon des dispositifs de présenta- 
tion et d'interprétation parfois similaires. Ils les stockent également selon des méthodes relativement identiques (afin d'en assurer la meilleure conservation). Les modes de valorisation de ces objets, cependant, varient considérablement entre les deux institutions. La question de la luminosité des espaces semble assez globalement départager les deux mondes. La lumière muséale, bien que contrainte (pour des raisons de conservation préventive), vise à apporter au public les conditions optimales de lisibilité des objets. C'est ce même principe qui guide la présentation des musealia: donner à voir, et donc présenter ces derniers afin qu'ils puissent être perçus sous leur meilleur jour, ou observés sous tous les angles. Les principes de monstration dans les temples ou les lieux de culte semblent reposer sur une logique inverse, selon laquelle l'obscurité révèle parfois mieux la dimension sacrée des objets. Les registres d'exposition du sacré semblent ainsi utiliser autant, sinon plus, l'ombre que la lumière, le passage au lumineux apparaissant comme une séquence plus limitée (comme l'ouverture d'un retable) que celle que l'on observe dans les musées. Le musée n'est pas totalement réfractaire à l'obscurité (qu'il utilise dans ses réserves), mais lorsqu'il recourt à cette dernière au sein de ses expositions, c'est soit pour plonger le spectateur dans le noir afin de mieux mettre en lumière les objets, soit (et plus récemment) pour évoquer le caractère sacré de certains objets spécifiques. Enfin, le choix des principes de monstration reflète, dans les deux cas, des hiérarchies de valeur très différentes. Si, pour une tradition religieuse spécifique, les critères d'exposition des objets sont liés à une hiérarchie inhérente au culte qui lui est propre, le musée se fonde sur des critères à la fois standardisés et égalitaires (les cultes et religions sont examinés à la lumière des connaissances scientifiques, tous les cultes étant égaux dans cette perspective) et hiérarchisés selon des critères patrimoniaux (l'ordre de présentation des objets est lié à leur valeur esthétique, historique ou leur apport scientifique).

\section{Le stade du musée}

La muséologie, au sens où Stránský l'entendait, suppose l'existence d'institutions antérieures aux musées tels que nous les connaissons : thesaurus, cabinets, etc. (Mairesse, 2019). Les exemples donnés par ce dernier n'intègrent pas les lieux de culte, le muséologue de Brno demeurant assez vague à cet égard. Il existe cependant depuis des siècles, dans ces endroits, des collections (œuvres d'art, mais aussi naturalia) plus ou moins liées au culte, que Lugli (1998) présente comme largement associés à l'origine des cabinets de curiosités. D’un point de vue historique donc, l'évolution conjointe des musées (ou de leurs prédécesseurs) et des lieux de culte mérite d'être observée. Un rapide coup d'œil sur l'évolution des deux institutions, en Occident, permet d'émettre l'hypothèse que l'évolution de l'un s'est produite parallèlement au déclin de l'autre, le nombre de musées créés semblant évoluer proportionnellement avec celui du nombre d'églises désaffectées (Mairesse, 20I4). 
Le glissement des objets et des espaces sacrés vers le profane (leur profanation), qui peut s'observer tout au long de l'histoire de l'humanité - effondrements de civilisations, comme l'Egypte, Rome, etc. mais aussi luttes et schismes, comme les vagues d'iconoclasme durant la Réforme, en Occident - a longtemps eu pour conséquence la disparition (ou l'enfouissement) de ces objets et de ces lieux spécifiques. Le développement du phénomène muséal, à partir de la Renaissance, suscite la création de tiers lieux, à mi-chemin entre le monde du sacré et celui des déchets inutiles, futur royaume de l'archéologue (Mensch, 1992). Au flux naturel des objets entre les deux mondes - espace du sacré, espace du profane puis du déchet - s'adjoignent dès lors de nouvelles circulations incluant les musées, mais aussi de nouvelles rencontres inédites entre les objets issus de ces différents registres. Longtemps considérés comme uniques témoignages d'un certain type de réalité, les voici soudain présentés comme autant de témoins, non d'une réalité mais d'une civilisation et de sa manière de concevoir le sacré. Le point de vue relativiste du musée présentant suspendus dans un même espace des artefacts issus de toutes les religions et croyances, a le mérite de développer un discours identique, construit sur un double registre scientifique et esthétique, mais au détriment des modes d'explication liés aux registres du sacré propre à chaque culte, lesquelles en ressortent en quelque sorte affaiblis, conduits à cohabiter ensemble à partir d'un discours unificateur mais réducteur. Il n'empêche que ces objets intégrés par les musées ont également permis de préserver (dans un état fantomatique ou plus ou moins comateux) des éléments - souvent organiques et périssables - qui, sinon, auraient largement risqué de disparaître.

C'est un tel mode d'appropriation du réel qui va progressivement être remis en question peu après la Seconde guerre mondiale, au gré des demandes de restitution puis, plus récemment, d'analyse critiques de la logique coloniale et prédatrice du musée. Au «musée prédateur », analysé par l'ICOFOM lors de la Conférence générale de Milan en 2016 (ICOFOM, 20I7) semblent répondre de nouvelles approches de l'institution, issues d'une muséologie moins fondée sur des valeurs d'objectivité scientifique que sur un processus de questionnement et de déconstruction, que l'on retrouve déjà dans la nouvelle muséologie française et la new museology britannique (Desvallées, 1992 \& 1994 ; Vergo, I989). Mais au profit de quel type de registre ? Le caractère prosélyte et hégémonique des religions monothéistes semble avoir déteint sur le monde muséal qui en est issu, et peut être critiqué en ce sens. A l'inverse, il est intéressant de noter la logique de cohabitation qui prévalait dans d'autres contextes, par exemple au sein de l'Empire romain dans lequel les cultes à Mithra ou à Isis pouvaient coexister avec la religion romaine (Scheid, 2008). Le renversement du «suprémacisme muséologique », tel que l'évoque Bruno Brulon Soares dans ce volume, laisserait-il la place à la coexistence pacifique entre plusieurs registres d'interprétation du sacré, ou à l'émergence d'autres discours tout aussi hégémoniques? 


\section{Approches du sacré au musée}

Les auteurs de ce numéro d'ICOFOM Study Series ont abordé le sacré de multiples manières. La thématique du musée occidental et colonisateur, rappelée plus haut, constitue une grille d'analyse sous-jacente à plusieurs articles : la notion du sacré, tel qu'il est présenté dans les musées occidentaux, reflète bien plus la vision des musées qu'elle ne témoigne des multiples aspects et des dimensions expérientielles du sacré dans d'autres régions du globe. Bruno Brulon Soares, en évoquant la muséologie comme l'expression d'une certaine foi, relève le caractère largement hégémonique de cette dernière, du moins dans les pays occidentaux. A partir de l'analyse de deux expositions liées au musée du Quai Branly, il souligne les limites du regard «scientifique » et esthétique de la muséologie francophone. Matías Cornejo González, prenant appui sur l'analyse du moai Hoa Hakananai'a de l'île de Pâques présenté au sein du British Museum, questionne à son tour le regard suprémaciste occidental, largement artistique, sur les cultures représentées, et la faiblesse des explications données sur les cérémonies associées aux objets, faisant de Rapa nui une culture vivante. Dans de telles conditions, comment comprendre l'essence même de ces objets de collection à travers ce type de regard? La présentation par Marion Bertin de l'organisation des réserves et des expositions au Vanuatu (répartition des réserves en fonction des interdictions, organisation du personnel à partir d'une connaissance et du respect des savoirs, organisation des expositions temporaires ou permanentes), témoigne a contrario des possibilités d'une autre muséologie (océanienne), et des différences considérables en matière d'organisation pratique des musées et du discours qui en résulte. Marília Xavier Cury évoque, à partir notamment de plusieurs exemples venus du Brésil, les possibilités de collaborations entre muséologues et autochtones, afin d'intégrer les connaissances ancestrales au sein du savoir muséal : autant de pistes pour une possible cohabitation.

Mais la relation au sacré apparaît tout aussi complexe pour les objets occidentaux. Fanny Fouché, à travers un examen du retable chrétien, « siège d'une rencontre possible entre immanence et transcendance », examine le caractère dévotionnel de ces ouvres et leur fonction de présentation de l'invisible à travers le visible, mais aussi le basculement progressif de la dévotion vers l'appréciation esthétique. «L'institution muséale est-elle en mesure de se porter garante aussi bien de la préservation matérielle de ce type d'objets que de la transmission du réseau hétérogène des raisons et significations multiples ayant fondé leur apparition? », s’interroge Fouché. Cette question résume les interrogations de la plupart des auteurs autour du rôle complexe de l'institution face aux enjeux de transmission des savoirs issus de civilisations et de sociétés multiples, parfois disparues ou en voie de disparition. Pour tenter de pallier ces difficultés, les professionnels des musées ont cherché à développer de nouveaux protocoles de préservation. Caitlin Spangler-Bickell, en examinant la manière dont sont utilisées les catégories d'inventaire et de conservation des autels et mausolées au sein du musée Fowler de UCLA, montre comment le personnel des musées cherche à préserver non seulement les aspects matériels, 
mais aussi les éléments éphémères donnant vie à ces formes sacrées et encore actives. Violette Loget et Yves Bergeron, à partir de l'évolution de la place de la religion au Québec, analysent la situation du patrimoine religieux dans cette province, les difficultés de financement que rencontrent les autorités ecclésiastiques, le rôle des pouvoirs publics et celui des musées. De nombreuses polémiques en lien avec la volonté par le clergé d'aliéner un certain nombre de ces biens, pourtant « hors commerce, incessibles et imprescriptibles » au sens de la loi du Bas-Canada, permettent d'évoquer le fait que les membres du clergé, parfois bien peu intéressés par la préservation des traditions, participent eux-mêmes à la dispersion de leur patrimoine, le rôle de la communauté muséale étant alors de lui rappeler ses devoirs en la matière. De manière plus générale, Helena Wangefelt Ström présente trois modèles permettant d'analyser la diversité de la place du sacré au musée et d'en analyser les conséquences : les modèles détaillant un spectre de possibilités allant de la destruction des identités précédentes d'un objet au profit de sa nouvelle identité muséale, à la possibilité d'utiliser de plusieurs manières les objets en les autorisant aussi bien à conserver leur caractère vivant qu'inanimé.

D'un point de vue plus institutionnel, Klas Grinell, à partir d'une analyse de l'UNESCO et de ses relations avec l'ICOM, examine le pouvoir sotériologique des musées, les envisageant en fonction du potentiel de «salut » portée par leur discours institutionnel : un salut apporté par le développement économique et la justice sur terre, l'éradication de la pauvreté et de la violence. De manière parallèle, Crispin Paine, dont on connait l'investissement autour des liens entre musées et religion, analyse le rapport au sacré, dans cet article, à partir de plusieurs institutions plus ou moins directement liées au champ muséal, évoquant les trésors d'église, les musées présentant des théories alternatives de l'évolution (créationnisme et sciences védiques) mais aussi un certain nombre de parcs à thèmes religieux (en Iran, en Inde, aux Etats-Unis, aux Pays-Bas ou au Japon).

Un tel éventail d'analyses n'appelle pas forcément de conclusions définitives quant à la place du sacré au sein du musée et la relation entre muséologie et sacré. Par le biais d'une phrase devenue célèbre, le muséologue britannique Kenneth Hudson résumait l'ambivalence de la présentation par le musée de la « réalité » : « un tigre empaillé dans un musée est un tigre empaillé dans un musée, et pas un tigre » (Hudson, I977, p. 7). De la même manière, comme le souligne également Jan Dolak dans son intervention, on se doit de reconnaître qu’un objet sacré dans un musée est un objet sacré dans un musée, et pas un objet sacré. Sa muséalisation ne permettra jamais - comme les tigres d'ailleurs - de restituer la même expérience que celle qui peut être vécue au sein d'un culte. En revanche, le musée, pour autant que l'on puisse faire le deuil de cette réalité vécue en d'autres lieux, constitue un espace de questionnement, de discussion et d'ouverture à d'autres réalités que le temple ne permet pas toujours. Cela pour autant qu'il renonce à la tentation d'imposer à son tour une vision globale et autoritaire, au nom de la science ou de l'esthétique, mais qu'il résiste aussi 
à la tentation de disparaître au profit du seul discours des autres : équilibre précaire donc, mais véritablement propice au dialogue et à la réflexion.

\section{Bibliographie}

Desvallées, A. (Ed.). (1992 \& I994). Vagues, une anthologie de la nouvelle muséologie. Mâcon : W \& MNES.

Duncan, A. \& Wallach, C. (1978). The Museum of Modern art as late capitalist ritual: an iconographic analysis, Marxist perspectives, 4, 28-5I.

Eliade, M. (1965). Le sacré et le profane. Paris : Gallimard.

Gregorová, A. (1980). La muséologie, science ou seulement travail pratique du musée ?, Mu Wop/ Do Tram, I, p. I9-2I.

Hudson, k. (1977). Museums for the 1980s. Paris: Unesco.

ICOFOM. (20I7). The predatory Museum, ICOFOM Study Series, 45.

Lugli, A. (1998). Naturalia et Mirabilia, Les cabinets de curiosité en Europe. Paris : Adam Biro.

Mairesse, F. (20I4). Le culte des musées. Bruxelles: Académie royale de Belgique (Académie en poche).

Mairesse, F. (2018). Museology and the Sacred. Materials for a Discussion. Paris: ICOFOM.

Mairesse F. (Ed.) (2019). Zbyněk Stránský et la muséologie. Une anthologie. Paris : L'Harmattan.

Mensch P. van (1992). Towards a Methodology of Museology. Zagreb: University of Zagreb, Faculty of Philosophy, Doctor's Thesis.

Otto, R. (1969). Le sacré. Paris : Payot.

Scheid, J. (2008). Pouvoir et religion à Rome. Paris : Larousse.

Texier, R. (I990). Sacré, in Jacob A. (ss la dir.) Encyclopédie philosophique universelle, II, Les notions philosophiques (pp. 229I-2293). Paris : Presses Universitaires de France.

Vergo, P. (1989). The New Museology. London: Reaktion Books. 